\title{
Study of Principal Catalogues of Visual Double Stars
}

\author{
Yikdem Mengesha Gebrehiwot ${ }^{1}$, Solomon Belay Tessema², Oleg Malkov² \\ ${ }^{1}$ Astronomy and Astrophysics Research Division, Entoto Observatory and Research Center (EORC), \\ Addis Ababa, Ethiopia \\ ${ }^{2}$ Institute of Astronomy, Russian Academy Sciences, Moscow, Russia \\ Email: yikdema16@gmail.com, tessemabelay@gmail.com, malkov@inasan.ru
}

Received 24 November 2015; accepted 17 December 2015; published 22 December 2015

Copyright (C) 2015 by authors and Scientific Research Publishing Inc.

This work is licensed under the Creative Commons Attribution International License (CC BY). http://creativecommons.org/licenses/by/4.0/

(c) (i) Open Access

\section{Abstract}

The goal of this study is to develop a tool for detection of errors and misprints in principal catalogues of visual double stars. Modern statistical investigations of visual doubles (which are about 130 thousand, and they represent the most numerous observational type of binaries) are based almost exclusively on data, listed in those catalogues. So their correction is a challenging task, otherwise the statistics of visual binaries can be biased. Study of individual stars should also be based on correct data. We have presented tools to detect some of errors in the catalogues, especially, to indicate cases i) when positional information on components of a pair is contradictory, and ii) when a double star or its component is included in a catalogue twice, under different names.

\section{Keywords}

Visual Binary Star, Positional Inconsistency, Data Duplication, Reference Coordinates, Additional Component Coordinates

\section{Introduction}

Heggie in his investigation of formation and evolution of binaries identified two classes of binary stars [1]. He called these binaries as "soft" and "hard" binary stars. Actually this classification is analogues to "wide" and "close" binary stars respectively. There is no well defined boundary between close and wide binary systems. It differs from author to author according to the purpose and types of the data required. The operative parameter of a binary system is the semi-major axis $a$. Since its values vary over a wide range of several orders of magnitude, the exact form of semi-major axis distribution is not well known yet. According to [2]-[4] binary stars can be 
more or less classified as "close" when semi-major axis $a \leq 10$ AU and "wide" when semi-major axis $a \geq 100$ AU.

Close binary systems are very essential to study stellar properties to a high certainty. One of the types of close binary systems, eclipsing binary systems, are used to determine stellar parameters like masses and radii of components of binary systems [5]. On the other hand, wide binary systems are essential systems to study star formation processes as well as an exceptionally useful tracer of local potential and tidal fields through which they traverse. Specifically, they have been used to place constraints on the nature of halo dark matter [6] and to explore the dynamical history of the Galaxy [7]. Moreover, wide binaries used to constrain the mass of hypothetical MAssive Compact Halo Objects, so-called "MACHOs" [8].

Visual binaries represent the most numerous observational types of binary stars. Catalogues of visual binaries contain more than $10^{5}$ pairs and provide us with astrometric, photometric and sometimes spectral information on the components. Consequently these data are extremely useful for statistical study and for construction of distributions on and relations between various observational and astrophysical parameters. However, catalogues of visual binaries are not error free. It is probably impossible to entirely fix the problem, but it is desirable to minimize the number of errors through various checks, to avoid any statistical investigations to be biased.

In this study we consider three principal catalogues of visual double stars, namely, The Washington Visual Double Star Catalog (WDS, [9]), the Catalog of Components of Double \& Multiple stars (CCDM, [10]), and the Tycho Double Star Catalogue (TDSC, [11]). They contain information on about 130 thousand (i.e., almost all known) visual double and multiple stars.

All catalogues contain annoying bugs and misprints, and this especially concerns large compilative catalogues WDS and CCDM. Some types of errors can be discovered without attraction of external data sources. These are, in particular, the cases (i) when positional information on an additional component in a pair is contradictory, and (ii) when a binary or a component is included in a catalogue twice, under different names.

Constructed tools and results of their application to the cases (i) and (ii) mentioned above are reported in Sections 2 and 3, respectively. Distribution of dublications as well as other errors found in the catalogues are discussed in Section 4. Finally, in Section 5 we make our conclusions.

\section{Positional Inconsistency in the Catalogues}

Catalogues of visual double stars provide us, among other data (brightness of the components, their spectral types, proper motion and cross-identification), with coordinates of the reference $\left(\alpha_{1}, \delta_{1}\right)$ and the additional $\left(\alpha_{2}, \delta_{2}\right)$ components of the pair. Also, the studied catalogues provide user with positional angle $(\theta)$ and separation ( $\rho$ ) of the additional component in a pair, i.e., its position relative to the reference component (with coordinates $\left.\alpha_{1}, \delta_{1}\right)$. From these data coordinates of the additional component $\left(\alpha_{3}, \delta_{3}\right)$ can also be calculated according to

$$
\begin{aligned}
& \alpha_{3}-\alpha_{1}=\frac{\rho \sin \theta}{\cos \delta_{1}} \\
& \delta_{3}-\delta_{1}=\rho \cos \theta,
\end{aligned}
$$

and compared with those, explicitely given in the catalogues $\left(\alpha_{2}, \delta_{2}\right)$, using a formula for calculation of angular distance between two points on the sky:

$$
d=\sqrt{\cos ^{2} \delta_{1}\left(\alpha_{2}-\alpha_{1}\right)^{2}+\left(\delta_{2}-\delta_{1}\right)^{2}} .
$$

It can be argued that if the distance $d$ exceeds certain selected value $d_{1}$, then the set $\left(\alpha_{1}, \delta_{1}, \theta, \rho, \alpha_{2}, \delta_{2}\right)$ contains inconsistent data. The value of $d_{1}$ depends on observational conditions, and should be estimated experimentally for every studied catalogue.

\subsection{WDS}

It should be noted that WDS (unlike the other two catalogues) does not provide coordinates for the additional component $\left(\alpha_{2}, \delta_{2}\right)$. It means that the detection of fictitious data may be performed only for those members of multiple (triple and higher multiplicity) systems, which appear as the reference component in one pair and as the additional component in another one. For example, WDS provides data for the following pairs of the system 
WDS 00013+6021: AB, AC, AD, BD, and the task can be performed, consequently, only for component $\mathrm{B}$.

The summary WDS catalog posted on-line provides two sets of $\theta, \rho$ values, for the first and the last observations (the full WDS database includes all measures). We used the latter one in this study.

For WDS we found $d_{1}=8$ arcsec, and there are about 340 pairs in the catalogue, demonstrating an inconsistency in positional data for additional components. The reasons for that inconsistency are large orbital motion in relatively close pairs (especially in a case of large difference in epochs of observation), large proper motion difference in optical pairs, confusion between two closely spaced objects, positional angle $\theta$ ambiguity by 180 degrees, large and consequently imprecisely defined value of separation $\rho$, misprints in the catalogue.

\subsection{CCDM}

CCDM, though being smaller than WDS, is based on a similar set of observational data (in fact it was based on an earlier version of the WDS). On the other hand, WDS is constantly updated, while CCDM keeps observational data collected before 2002. Also, unlike WDS, the detection of fictitious data may be performed for all catalogued objects (as coordinates are provided for every component in CCDM). That is why when we set $d_{1}$ for CCDM to be 8 arcsec, we found 1070 pairs demonstrating inconsistency between catalogued secondary coordinates and ones, calculated from primary coordinates, positional angle and separation.

Here we do not consider pairs, where $\theta$ is indicated roughly in CCDM, in the format like "SF" (to the South and following, i.e., second quadrant) or "NP" (to the North and preceding, i.e., fourth quadrant). Note that there are also pairs in the WDS with $\theta$ only published as N, S, SF, NP, etc. These have been converted to coarse $\theta$ values such as $0,45,90$, etc. Usually these quadrant-only position angles were published only for very early measures, so the "last" angles are nearly always more accurately measured values.

\subsection{TDSC}

TDSC is a homogeneous catalogue, and it contains observations performed with the same instrument. As a result for the majority of objects we found $d_{1}$ to be about 1.2 arcsec. This limit is exceeded by eight very wide ( $\rho$ exceeds 10 arcmin) pairs only. For the extreme case $d=27.4$ arcsec (TDCS $56356=$ WDS 20452$3120 \mathrm{AB}, \rho=78$ arcmin).

\section{Data Duplication in the Catalogues}

Some star can happen to be a member of two different double (multiple) systems, included in a compilation catalogue of doubles. Moreover, a double star can be discovered by two different observers independently and, consequently, this pair appears twice in the catalogue, under different designations. This can be checked by coordinate comparison. Similarly to the case described in Section 2, angular distance between two points with celestial coordinates $\left(\alpha_{x}, \delta_{x}\right)$ and $\left(\alpha_{y}, \delta_{y}\right)$ can be calculated according to

$$
d=\sqrt{\cos ^{2} \delta_{x}\left(\alpha_{y}-\alpha_{x}\right)^{2}+\left(\delta_{y}-\delta_{x}\right)^{2}}
$$

and compared to certain limiting value $d_{2}$. If $d$ does not exceed certain $d_{2}$, one can conclude that $\left(\alpha_{x}, \delta_{x}\right)$ and $\left(\alpha_{y}, \delta_{y}\right)$ correspond to the same celestial object.

Again, $d_{2}$ value depends on observational conditions, and we should estimate it experimentally for every studied catalogue. Preliminary results were checked manually with the Binary star database (BDB, [12]), which allows us to visualize catalogued data, and SIMBAD.

\subsection{WDS}

As our analysis shows, $d_{2}$ (WDS) value is 21 arcsec, and all (altogether 306) cases with $d<21$ arcsec indicate duplication of objects in WDS. Beside that, 26 other duplication cases are found, and they exhibit coordinate difference to be $21<d<41$ arcsec. At the same time we have found 59 cases when the angular distance between two really different objects is in the $21<d<41$ arcsec range.

The absence of a strict border between these two cases can be explained by variations of the positional accuracy of the catalogued data. In some cases coordinates of the objects are known with relatively low preci- 
sion. It is true for overcrowded areas (e.g., Orion nebula), for very bright (e.g., $\alpha$ Cap) or very faint (e.g., WDS 19252-2900) stars, and for relatively wide $(\rho>200$ arcsec) pairs (e.g., WDS 09479+1319). It also happens when K-band or other infrared magnitudes are given in WDS (e.g., vicinity of RK86 reflection nebula) instead of the visual photometry.

Duplication cases can be classified according to the following scheme.

- A star is included in two different double/multiple systems. E.g., WDS 00086-0418 A = WDS 00085-0419 A (Figure 1).

- Both components of a catalogued double star are included in another, also catalogued, multiple system. Those two stars can constitute (e.g., WDS $23243+6216=$ WDS $23248+6217$ CD, Figure 2) or cannot constitute (e.g., WDS $23288+0703$ A and B are WDS $23287+0703$ C and B, respectively, Figure 3) a pair in the multiple system.

- Two catalogued double stars represent in fact the same pair of objects (Figure 4 and Figure 5). E.g., WDS 00281-2652 AB - WDS 00280-2654 AB.

All of these cases should be taken into account in statistical research of visual binaries. We have reported on these errors to the WDS authors, and necessary corrections were made in the WDS catalogue.
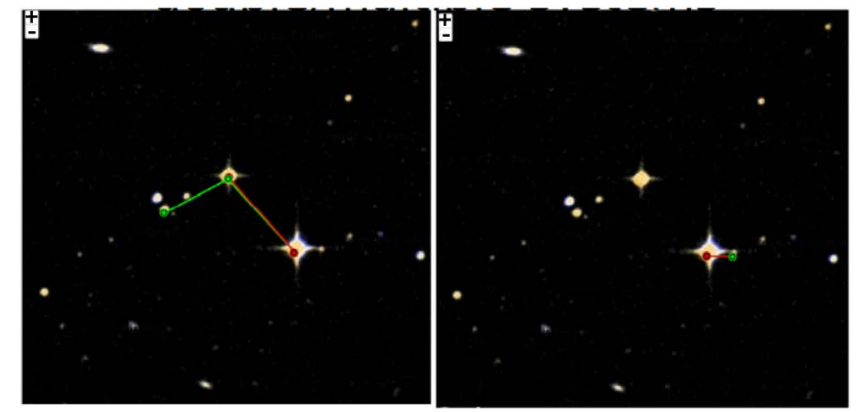

Figure 1. WDS 00086-0418 and WDS 00085-0419. Duplication case 1, a star is included in two different double/multiple systems.
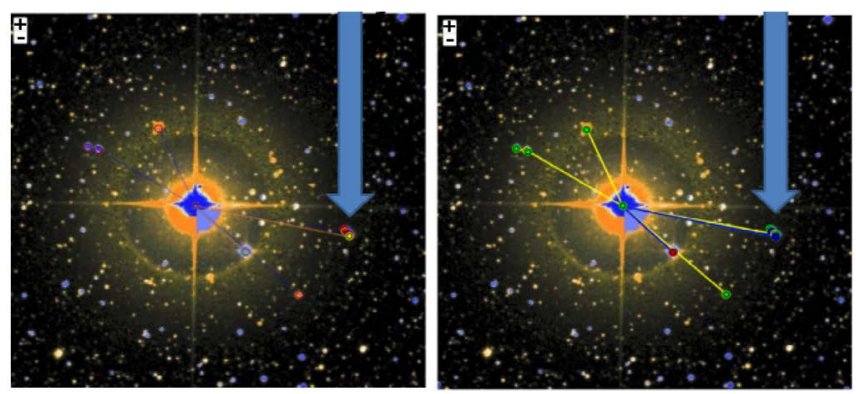

Figure 2. WDS $23243+6216$ and WDS $23248+6217$. Duplication case 2a, a catalogued double star are included in another system.
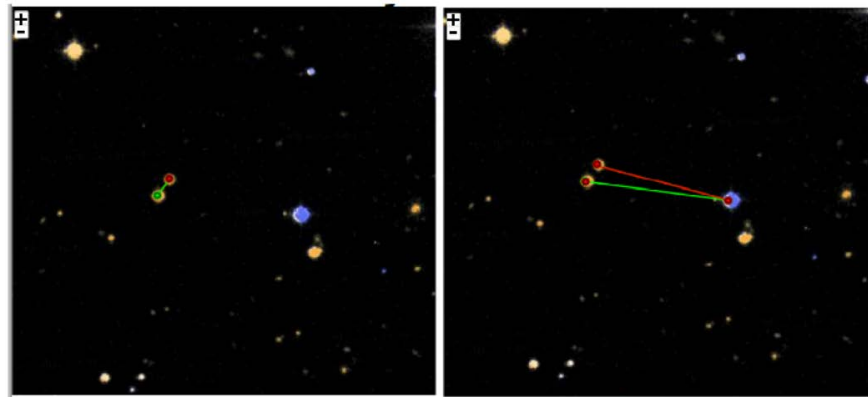

Figure 3. WDS 23288+ 0703 and WDS 23287 + 0703. Duplication case 2b, A catalogued double star is included in another system. 

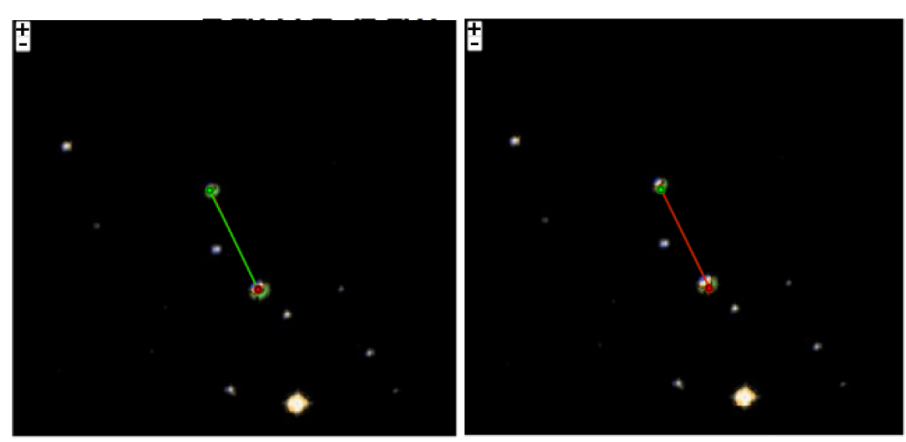

Figure 4. Duplication case 3. Two catalogued double stars are in fact the same pair of objects.
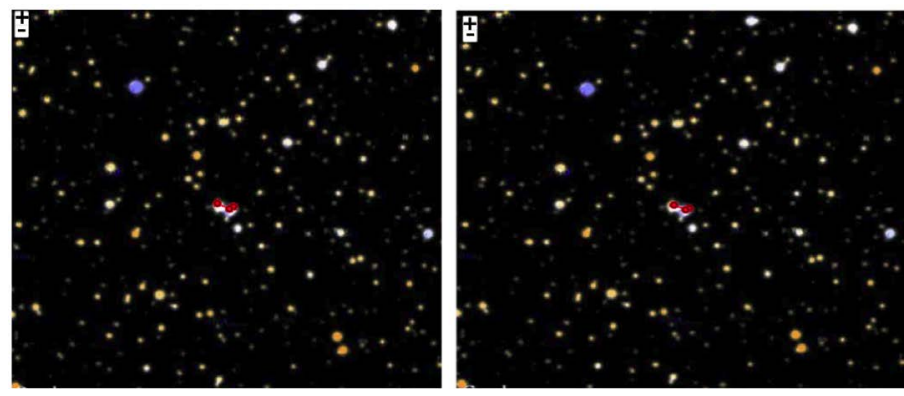

Figure 5. WDS $01450+6032$ and WDS $01450+6038$. Duplication case 3, two catalogued triple systems are the same.

\subsection{CCDM}

For CCDM we estimate $d_{2}$ to be about 11 arcsec, and in 19 cases components are included in two different binary/multiple systems in the catalogue (Figure 6 and Figure 7).

\subsection{TDSC}

Owing to its homogeneity, we did not find duplications in TDSC, at least at the level of $d_{2} \approx 50$ arcsec. The only exception is TDSC $29583 \mathrm{~A}=$ TDSC $29584 \mathrm{~A}$.

\section{Discussion}

\subsection{Data Duplication}

It is instructive to compare distributions of duplication cases found in Sections 3.1 and 3.2 on component separation $\rho$. They are shown in Figure 8 and Figure 9, respectively. One can see that the maximum of the WDS distribution is shifted to the smaller $\rho$ in comparison with thr CCDM distribution, as WDS includes more modern and more precise observations and, consequently, can resolve closer pairs. Note also the difference of Y-axis scale in Figure 8 and Figure 9.

\subsection{Other Errors in the Catalogues}

Sometimes catalogued positional angle $\theta$ can be ambiguous by 180 degrees. This is not always observer"s error. In some cases magnitude difference is too small for the observer to tell. It may also depend on the observing technique used- if the stars have different colors, the brighter star in B may not be the brighter star in R. Some of such cases can be indicated in WDS. As it was mentioned above, WDS provides two sets of $(\theta, \rho)$ values, for the first and the last observations. So, if the difference between $\theta$ values, obtained at the first and at the last observations, is close to 180 degree, it looks questionable. One can select pairs, where difference between 180 degree and $\left|\theta_{\text {last }}-\theta_{\text {first }}\right|$ does not exceed some $d_{3}$. To avoid pairs with high orbital motion, one 

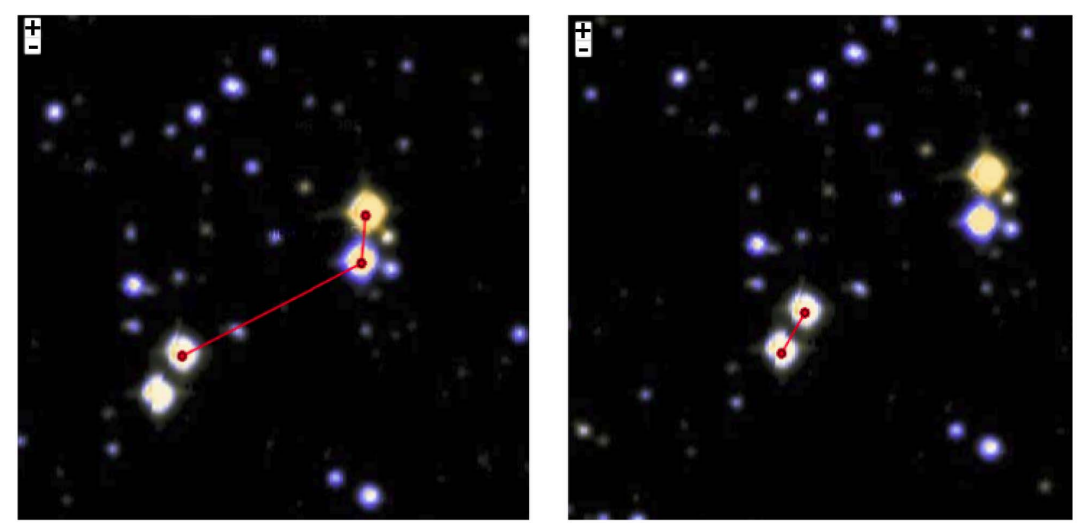

Figure 6. CCDM $00188+5924$ C and CCDM $00189+5923$ A, Duplication case 1 . A star is included in two different double/multiple systems
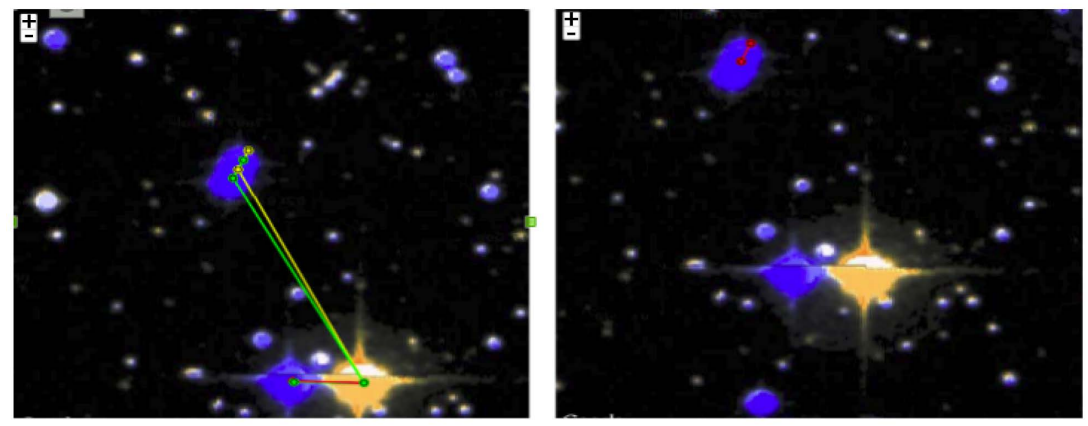

Figure 7. CCDM 01460 + 7142C and CCDM 01461 + 7143A, Duplication case 2. A catalogued double star is included in another system.

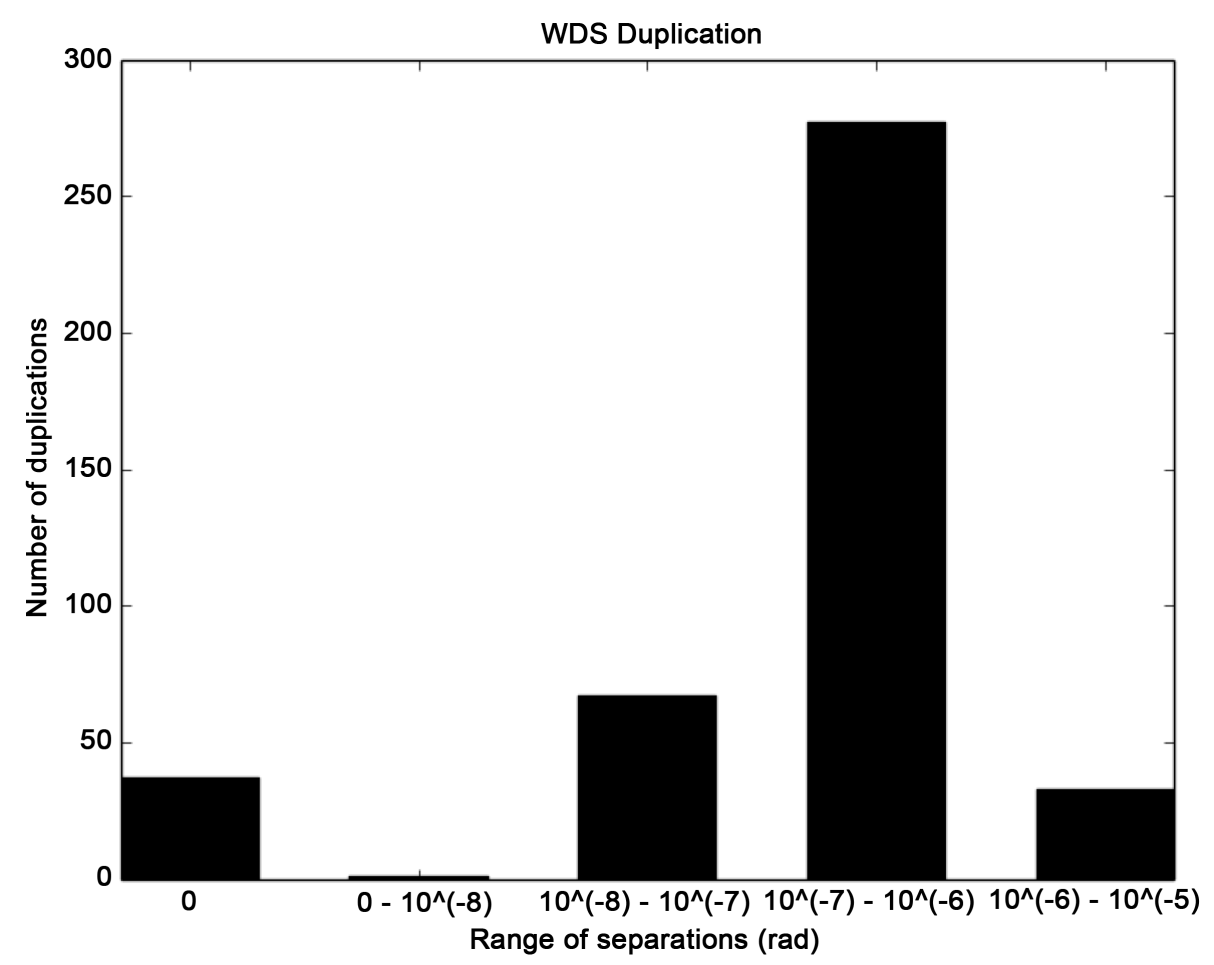

Figure 8. Distribution of duplications in WDS on the separation. 


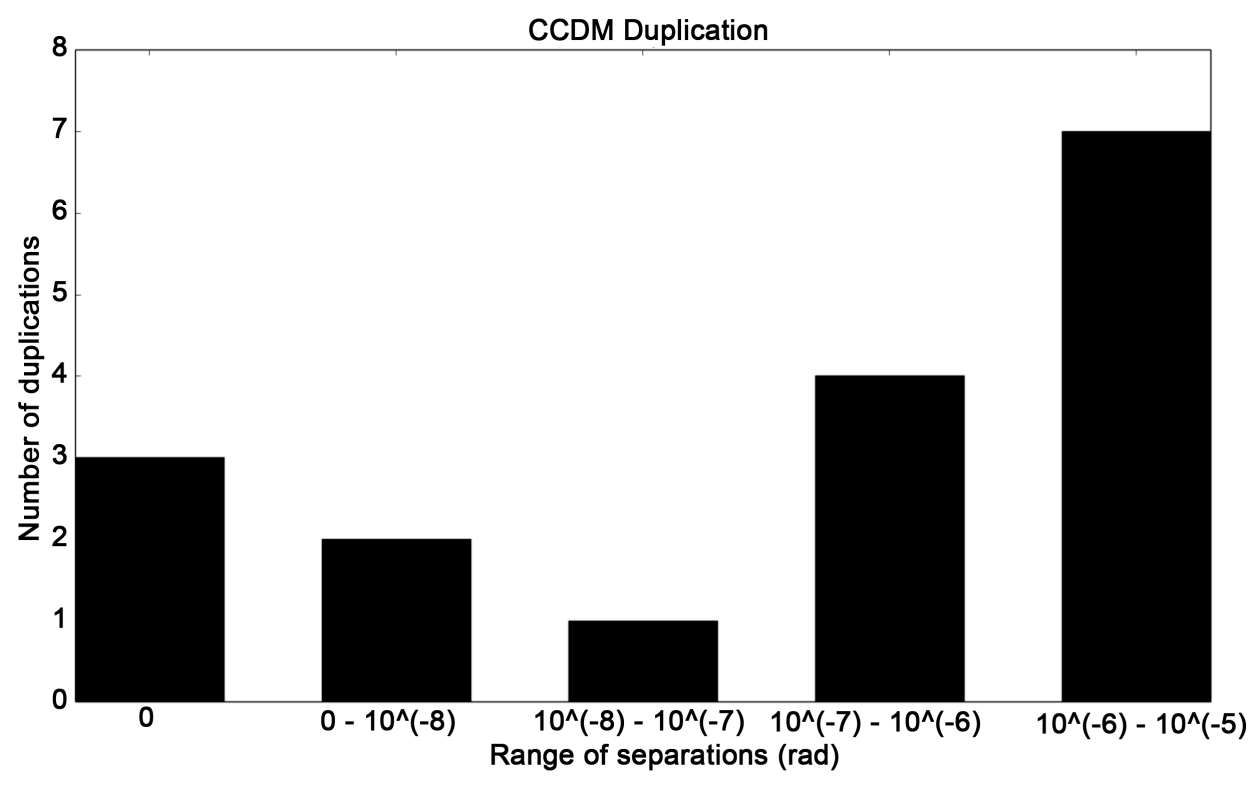

Figure 9. Distribution of duplications in CCDM on the separation.

can consider only pairs with $\rho>10$ arcsec. If one takes $d_{3}=15$ degree, the resulting list contains 72 entries. Ten of them are happened to be pairs with large proper motions differences (and consequently with correct catalogued data), but other pairs contain erroneous data. To make the estimations more reliable, observation epoch difference can be taken into account.

All errors found in WDS were reported to the authors of this constantly updated catalogue.

TDSC contains cross-identification with WDS, but we have found that TDSC is apparently incorrect in identifying some objects with WDS objects, and, consequently, at least parts of the WDS names listed in TDSC are not correct. So we urge to be wary of WDS identifiers in TDSC. We note that TDSC contains data on 64894 systems, despite the fact that the latter number is 65210 .

It should be noted also that our analysis has revealed about 70 errors (misprints) in CCDM, and the list of the errors is submitted to VizieR.

\section{Conclusions}

In this paper we developed methods of detection of errors in catalogues of double stars and presented results of their application to three principal catalogues of visual double and multiple stars, WDS, CCDM and TDSC.

Reports on the errors, detected in the studied catalogues, were sent to authors of the catalogues or to VizieR database. In some cases we could indicate a reason for the detected errors and suggest a way for correction. All lists of detected errors are available upon request.

\section{Acknowledgements}

We are grateful to William Hartkopf for helpful discussion and suggestions. We acknowledge the help of Negessa Tilahun, Seblu Humne, Tesfaye Dagne and Zeleke Amado. We thank Entoto Observatory and Research Center Astronomy and Astrophysics Research Division for giving such an opportunity and research facilities, and Mekele University for partly supported the research. This work was also partly supported by the Russian Foundation for Basic Researches (grants 15-02-04053 and 16-07-01162), by the Program of fundamental researches of the Presidium of RAS (P-41) and by the Program of support of leading scientific schools of RF (3620.2014.2). The use of the SIMBAD and WDS databases are acknowledged.

\section{References}

[1] Heggie, D.C. (1975) Binary Evolution in Stellar Dynamics. MNRAS, 173, 729-787.

http://dx.doi.org/10.1093/mnras/173.3.729 
[2] Luyten, W.J. (1979) New Luyten Catalog of Stars with Proper Motions Larger than Two Tenths of an Arcsecond. Minneapolis: Univ. of Minnesota Press.

[3] Chanamé, J. and Gould, A. (2004) Disk and Halo Wide Binaries from the Revised Luyten Catalog: Probes of Star Formation and MACHO Dark Matter. The Astrophysical Journal, 601, 289. http://dx.doi.org/10.1086/380442

[4] Salim, S. and Gould, A. (2003) Improved Astrometry and Photometry for the Luyten Catalog. II. Faint Stars and the Revised Catalog. The Astrophysical Journal, 582, 1011. http://dx.doi.org/10.1086/344822

[5] Andersen, J. (1991) Accurate Masses and Radii of Normal Stars. The Astronomy and Astrophysics Review, 3, 91-126. http://dx.doi.org/10.1007/BF00873538

[6] Yoo, J., Chanamé, J. and Gould, A. (2004) The End of the MACHO Era: Limits on Halo Dark Matter from Stellar Halo Wide Binaries. The Astrophysical Journal, 601, 311. http://dx.doi.org/10.1086/380562

[7] Allen, C., Poveda, A. and Hernańdez-Alcańtara, A. (2007) Halo Wide Binaries and Moving Clusters as Probes of the Dynamical and Merger History of our Galaxy. In: Hartkopf, B., Guinan, E. and Harmanec, P., Eds., Binary Stars as Critical Tools and Tests in Contemporary Astrophysics, Proceedings of IAU Symposium No. 240, Cambridge Univ. Press, Cambridge, 405.

[8] Alcock, C., Allsman, R.A., Alves, D.R., Axelrod, T.S., Becker, A.C., Bennett, D.P., et al. (2001) MACHO Project Limits on Black Hole Dark Matter in the 1 -30 M Range. The Astrophysical Journal Letters, 550, L169. http://dx.doi.org/10.1086/319636

[9] Mason, B.D., Wycoff, G.L., Hartkopf, W.I., Douglass, G.G. and Worley, C.E. (2014) VizieR On-line Data Catalog: B/wds.

[10] Dommanget, J. and Nys, O. (2002) VizieR On-line Data Catalog: I/274.

[11] Fabricius, C., Høg, E., Makarov, V., Mason, B., Wycoff, G. and Urban, S. (2002) The Tycho Double Star Catalogue. Astronomy \& Astrophysics, 384, 180-189. http://dx.doi.org/10.1051/0004-6361:20011822

[12] Kovaleva, D.A., Kaygorodov, P.V., Malkov, O.Yu., Debray, B. and Oblak, E. (2015) Binary Star DataBase BDB Development: Structure, Algorithms, and VO Standards Implementation. Astronomy \& Computing, 11, 119-125.

http://dx.doi.org/10.1016/j.ascom.2015.02.007 